\title{
Infrared and ultraviolet laser removal of crustose lichens on dolomite heritage stone
}

Mikel Sanz ${ }^{1}$, Mohamed Oujja ${ }^{1}$, Carmen Ascaso $^{2}$, Asunción de los Ríos ${ }^{2}$, Sergio PérezOrtega $^{2}$, Virginia Souza-Egipsy ${ }^{3}$, Jacek Wierzchos ${ }^{2}$, Mariela Speranza ${ }^{2}$, Maria Vega Cañamares $^{4}$, Marta Castillejo ${ }^{1 *}$

${ }^{1}$ Instituto de Química Física Rocasolano (IQFR), CSIC, Serrano 119, 28006 Madrid, Spain

${ }^{2}$ Museo Nacional de Ciencia Naturales (MNCN) CSIC, Serrano 115 bis, 28006 Madrid, Spain

${ }^{3}$ Instituto de Ciencias Agrarias (ICA), CSIC, Serrano 115 bis, Madrid 28006, Spain ${ }^{4}$ Instituto de Estructura de la Materia (ICEM), CSIC, Serrano 121, Madrid 28006, Spain *Email: marta.castillejo@iqfr.csic.es

\begin{abstract}
Laser removal of biodeteriogen layers warrants detailed studies due to the advantages it brings with respect to mechanical elimination or the use of biocides. We have investigated elimination of biological crusts on dolomite stones from heritage sites in central Spain. The samples were colonized by epilithic crustose lichens of different species, such as Caloplaca sp. and Verrucaria nigrescens. A comparative study was carried out by
\end{abstract}


applying infrared $(1064 \mathrm{~nm})$ and ultraviolet $(355 \mathrm{~nm})$ nanosecond laser pulses and sequences pulses of the two wavelengths using a Q-switched Nd:YAG system. To detect anatomical and ultrastructural damage to the lichens, and to assess possible morphological and chemical changes on the underlying stone induced by laser irradiation, we used stereomicroscopy, scanning electron microscopy with backscattered electron imaging and Fourier transform Raman spectroscopy. The optimal conditions for removal of the colonization crust, while ensuring preservation of the lithic substrate, were obtained for dual infrared-ultraviolet sequential irradiation.

Keywords: Laser removal, biodeterioration, biological crust, dolostone, lichens, photobiont, mycobiont.

\section{Highlights}

- Laser irradiation at $1064 \mathrm{~nm}$ (IR) or $355 \mathrm{~nm}$ (UV) partially removes epilithic lichens on dolostone.

- Irradiation in a sequential, dual IR-UV mode efficiently eliminates lichen thalli.

- Dual IR-UV irradiation mode induces severe damage on endolithic colonizers of dolostone.

\section{Introduction}

Architectural stones exposed to environmental pollutants and an array of past conservation treatments, including the application of consolidants, often exhibit a condition where a hard crust of dirt and contamination is well adhered to the stone and the protecting outer layers of the stone substrate. In certain critical cases, traditional techniques of microsand 
blasting, chemical poultices and water washing have failed to protect the fragile lithic substrate. Lasers constitute a promising alternative to more conventional cleaning techniques for certain applications [1-7]. In fact, laser cleaning of stone is a wellestablished procedure because it provides fine and selective removal of superficial deposits and encrustations. This has been demonstrated in a number of laboratory and on-site conservation projects, $[3,5,8]$. In particular, irradiation with nanosecond (ns) pulses of $1064 \mathrm{~nm}$ efficiently removes black encrustations, although yellowing of the stone substrate often accompany the cleaning process. To correct and/or prevent such undesired coloration, several alternatives have been proposed, including the use of (infrared (IR) and ultraviolet (UV) radiation, both in sequential and synchronous mode [4,8-10], and the use of laser pulses of different duration, in the range of nanoseconds-microseconds [5].

Stone used in heritage buildings and monuments is prone to host lithobiontic communities [11-13]. These can be constituted by diverse microorganisms, such as heterotrophic bacteria, cyanobacteria, free-living algae and fungi, and by lichens. Lichens are a symbiotic association of a fungus (the mycobiont) with a photosynthetic partner (the photobiont, an alga and/or cyanobacteria). These lithobiontic microorganisms colonize not only the surface of the lithic substrate (epilithic colonization), but also the bulk (endolithic colonization) where they develop complex interactions with the rock minerals $[14,15]$. Such interactions derive in physicochemical modifications which eventually enhance weathering reactions and lead to deterioration of the rock substrate. Remediation protocols for stone degradation by biological attack include the use of biocides or sterilization by UV light, but their effect is often limited to the surface of the material treated [16-18] and, in the case of biocides, irreversible damaging side effects may be induced on the rock materials. 
Laser removal of biodeterioration films from stone has been investigated in foregoing studies [19-23]. The action of the Er:YAG laser, operating at $2.94 \mu \mathrm{m}$, has been tested for the removal of lichens on limestone [20]. Elimination of biological crust from granite was studied using nanosecond laser pulses of $355 \mathrm{~nm}$ [21]. In a previous work [22], we have reported on the control of biodeterioration processes induced on dolostone by the lichen species Verrucaria nigrescens using a Q-switched ns Nd:YAG laser at $1064 \mathrm{~nm}$. In this case, laser irradiation caused the inactivation of endolithic microorganisms and the partial removal of epilithic colonizers. Recently, Osticioli et al. [23] have compared the effect of pulse duration and wavelength of Nd:YAG laser in the elimination of $V$. nigrescens from Carrara marble substrates and have shown the advantages of irradiation at $532 \mathrm{~nm}$. These previous investigations have shown that the laser effects on biological encrustations vary with the type of treated lithobiontic communities and with the petrographic and mineralogical characteristics of the substrate. Therefore, research aiming at investigating and characterizing laser parameters for treatment of biological encrustations, while ensuring safe preservation of heritage stone substrates, is of high interest for profiting of the advantageous use of laser methods in the conservation of monuments and historical buildings.

Herein, we have investigated the laser removal of epilithic crustose lichens colonizing dolomite stones from central Spain, the mentioned $V$. nigrescens on historical quarry dolostone and unhealthy Caloplaca sp. on monumental dolostone. To that purpose we have applied a methodology successfully developed for the removal of black crust on stone [4,9], based on sequential IR-UV laser irradiation. We compared the removal of biolayers and the effects induced on the lithic substrate by irradiation with IR, at $1064 \mathrm{~nm}$, and UV, at $355 \mathrm{~nm}$, laser pulses, and with sequences of IR followed by UV pulses. To that purpose we used the fundamental $(1064 \mathrm{~nm})$ and 3rd harmonic $(355 \mathrm{~nm})$ output of a ns Q-switched 
Nd:YAG system. Then, to evaluate the structural and physiological damage inflicted on the lichens upon laser irradiation, and also to assess possible modifications on the underlying stone, we used several analytical techniques. Stereomicroscopy served to appraise structural changes of the biological crust, while cross sections of the treated samples were observed by scanning electron microscopy with backscattered electron imaging (SEMBSE). With this technique it becomes possible to identify lichen anatomical changes and cytological symbiont effects. Fourier transform (FT) Raman spectroscopy was employed to identify possible structural and chemical changes on irradiated zones of the lithic substrates. Dolomite is a stone type widely used for the construction of heritage buildings; therefore the results derived from this study are of great significance in future planned conservation campaigns aiming at the control of biodeterioration layers on heritage buildings and stone monuments using lasers and/or biocides.

\section{Experimental}

For this study we selected two dolomite samples for laser treatment. The first one is a monumental sample from Segovia, partially colonized by epilithic crustose thalli of the lichen Caloplaca sp. forming a brown crust of about $500 \mu \mathrm{m}$ thick. Lichen thalli were not found in a perfect health condition in this sample, likely due to anthropic effects caused by its localization in the monument. The second sample is a dolostone fragment from an historical quarry in Redueña, Madrid. The surface of this sample is covered by encrustations of the also epilithic crustose lichen $V$. nigrescens. The thalli in this case are about the same thickness as those of Caloplaca sp. of the first sample. However, in $V$. nigrescens the thalli are darker and display much crowded areoles. We applied sequences of laser pulses to hydrated sample areas with lichen thalli that were selected after monitoring the surface with a Leica EC3 stereomicroscope equipped with a NIKON 
COOLPIX 8800 VR camera. Steromicroscopy was also used to detect surface changes upon irradiation. The samples were maintained under controlled low humidity conditions during irradiation and measurements. Laboratory irradiation tests were carried out with a Q-switched Nd:YAG laser (pulse width $15 \mathrm{~ns}$, repetition frequency $1 \mathrm{~Hz}$ ) using the fundamental wavelength of 1064 and its third harmonic at $355 \mathrm{~nm}$. We applied sequences of pulses at either individual IR or UV wavelengths or sequences of IR followed by UV pulses under the same irradiation path. The unfocussed laser beams, with a cross section of ca. $0.25 \mathrm{~mm}^{2}$, were directed to the surface of the sample with the help of mirrors. During irradiation the samples were translated perpendicularly to the laser propagation direction to obtain uniformly irradiated areas of up to $1 \mathrm{~cm}^{2}$.

After laser irradiation and scrutiny by steromicroscopy, the stone samples were prepared for SEM-BSE observation using the method developed by Wierzchos and Ascaso [24]. This technique allows for the fine monitoring of the action of the laser at cytological level and for the assessment of the structural modifications of epilithic and endolithic growths. In short, a fine-polished surface of a cross-section of the non-irradiated and irradiated zones was carbon coated and examined using a FEI Inspect S SEM equipped with a fourdiode, semiconductor BSE detector. The microscope operated at a tilt angle of $0^{\circ}$, acceleration potential of $15 \mathrm{kV}$, working distance of $15 \mathrm{~mm}$ and specimen current range of 1-5 nA.

Together with microscopy, FT-Raman spectroscopy was employed to detect possible structural and chemical changes on the irradiated zones. We used a RFS 100/S-G Bruker spectrometer equipped with a cooled Ge detector. The excitation source consists of a continuous Nd:YAG laser emitting at $1064 \mathrm{~nm}$. We applied low laser power outputs (10-20 $\mathrm{mW}$ ) to ensure absence of damage to the samples during measurements. The light scattered from an area of $<0.01 \mathrm{~cm}^{2}$ was collected in backscattering $\left(180^{\circ}\right)$ geometry. Each data 
point resulted from the accumulation of 200 scans and the wavenumber resolution was 4 $\mathrm{cm}^{-1}$. All FT-Raman spectra were baseline corrected.

\section{Results}

With the help of visual and steromicroscopic observations we defined the laser conditions for removal of the colonization crusts while ensuring absence of damage or fracture of the dolostone substrates. For the samples considered here we irradiated with sequences of 100 pulses of $1064 \mathrm{~nm}$ at a fluence of $2.5 \mathrm{~J} \mathrm{~cm}^{-2}$. At $355 \mathrm{~nm}$ we used the same number of pulses at a fluence of $0.5 \mathrm{~J} \mathrm{~cm}^{-2}$. These fluences are below the corresponding bare stone ablation thresholds, which were determined as reported in [9] and to be $3.0 \pm 0.1$ and 0.60 $\pm 0.03 \mathrm{~J} \mathrm{~cm}^{-2}$ at 1064 and $355 \mathrm{~nm}$ respectively. For dual IR-UV sequential irradiation we applied 100 IR pulses followed by 100 UV pulses of the indicated fluences.

By means of stereomicroscopy we screened structural modifications of the biological crust and by using SEM-BSE we were able to monitor the changes in the anatomy of the epitlithic lichen thalli and the morphological alterations in the photobiont and mycobiont cells induced under the different laser irradiation conditions.

Figures 1 and 2 illustrate the effect of IR irradiation on the Caloplaca sp. lichen crust of the dolostone sample from Segovia. Specifically, Fig. 1a shows a steromicroscopy image of an area of the sample irradiated with 100 pulses of $1064 \mathrm{~nm}$ at $2.5 \mathrm{Jcm}^{-2}$. The laser treatment induces a superficial change of colour of the crust to a lighter tone as already reported in [22]. Fig. 1b displays a SEM-BSE image of Caloplaca sp. thalli and the associated endolithic colonization of this sample in a cross section at the boundary between a non-irradiated and irradiated area (to the right and left of the arrow respectively). This image places in evidence the removal of lichen areoles by the action of the laser. 
In Fig. 2a, corresponding to the non-irradiated area of the same sample, the upper cortex (asterisk) and photobiont layer (between the two dotted lines) of the non-irradiated unhealthy Caloplaca sp. lichen thalli are distinguished. The effect of IR laser irradiation on the biological crust is demonstrated in Fig. 2b. Epilithic thalli have been partially removed and microbial cells localized near the stone surface, appear damaged in the irradiated area. This is evident by the presence of cell wall remains and absence of cellular protoplast, visualized as black empty spaces, instead of the living cells of the non-irradiated area, observed in Fig. 2a. The lichen crust in this sample is not totally removed at the present irradiation conditions. Higher laser fluences would be required for this purpose, but at the expense of inducing ablation and/or damage of the underlying stone.

We studied the effects of UV laser irradiation by considering the dolostone sample from Redueña quarry. Figures 3-5 summarize the results obtained. The stereomicroscopy image of Fig. 3a shows the non-irradiated sample surface covered by the dark-brown areolate thalli of $V$. nigrescens. A cross sectional SEM-BSE image (Fig. 3b) offers detail of the biological crust harbouring $V$. nigrescens thalli and endolithic colonization before irradiation. The upper cortex, the photobiont layer and the lichen medulla are clearly distinguished in the image. In Fig. 4a, the SEM-BSE image of a non-irradiated area shows the upper cortex and the algal layer at higher magnification. Fig. $4 \mathrm{~b}$ displays a stereomicroscopy image of an area irradiated with 100 pulses of $0.5 \mathrm{~J} \mathrm{~cm}^{-2}$ at $355 \mathrm{~nm}$. Comparison of this image with the one of a non-irradiated area of this sample, as shown in Fig. 3a, reveals the partial removal of the lichen crust. This is put in evidence by the change of colour from the initial dark tone of the lichen thalli (Fig. 3a) to the green coloration of the areoles of the remaining thalli (Fig. 4b), as laser irradiation of the thallus induces the exposure of algal cells within the algal layer by structural alteration of the upper cortex. This alteration is observed in detail in the SEM-BSE image of Fig. 5a where 
lichen areoles appear partially destroyed in the treated areas. Further insight of the effects of UV irradiation are presented in Fig. 5b which shows that in some areas the upper cortex is partially removed (arrow) and the fungal cortex cells appear plasmolyzed (asterisks). Fig. 5 clearly reveals that UV laser irradiation has a rather superficial effect on the lichen crust, in variation with the more in-depth effect observed for IR laser treatment (see above and previous results in [22]).

Effects of sequential IR-UV irradiation are illustrated by the results presented in Figures 6 and 7 , which refer to the dolostone sample from Redueña quarry. The stereomicroscopy image (Fig. 6a) was obtained after sequential IR-UV laser treatment with 100 pulses of $1064 \mathrm{~nm}$ at $2.5 \mathrm{~J} \mathrm{~cm}^{-2}$ followed by 100 pulses of $355 \mathrm{~nm}$ at $0.5 \mathrm{~J} \mathrm{~cm}^{-2}$. Through the comparison of this image with that of a non-irradiated area of the sample (Fig. 3a), it is possible to identify regions where several $V$. nigrescens areoles have been completely removed by laser irradiation and to appreciate the final lighter coloration of the irradiated rock surface. Fig. 6a also reveals the change of colour of the remains of areolated thalli, from the initial dark brown to a green pigmentation. Therefore it becomes clear that the effects of dual IR-UV laser irradiation seem more dramatic for epilithic lichen colonization than the laser treatment based in the application of only IR pulses. The broken line in Fig. 6a encircles a region of the irradiated area, as described above, which underwent further observation under the electron microscope in SEM-BSE mode. The effect of dual laser irradiation is demonstrated in Fig. 6b, where complete removal of the lichen areoles becomes evident and the damaging effects inflicted on microbial cells below the surface, are witnessed by the observed remains of fungal hyphae. This confirms that the dual laser irradiation scheme, not only efficiently removes the lichen crust, but also strongly damages the endolithic colonizers. Images in Fig. 7 provide evidence of the laser damage on lichen areoles that have not been totally removed by irradiation. Figs. 7 a,b show how the upper 
lichen cortex has been partially destroyed, leaving exposed algal cells (wide arrow in Fig. 7b) which have experienced the loss of some of their content (asterisks in Fig. 7b).

Finally we analyzed by FT-Raman spectroscopy the non-irradiated stone control areas and the areas where the laser treatment served for partial or complete elimination of the biodeteriogen film. Spectra are shown in Figure 8. The black line corresponds to the spectrum of the bare dolomite stone, acquired in a lichen-free, back side of the sample. The bands at $175,299,727$ and $1097 \mathrm{~cm}^{-1}$ are attributed to the vibration modes of the free $\mathrm{CO}_{3}{ }^{2-}$ ion of $\mathrm{CaMg}\left(\mathrm{CO}_{3}\right)_{2}$ (dolomite). In particular the bands at 175 and $299 \mathrm{~cm}^{-1}$ are assigned to lattice translational modes and those at 727 and $1097 \mathrm{~cm}^{-1}$ to in-plane bending and symmetric stretching modes of the carbonate ion respectively [25]. The spectrum of a sample zone colonized by $V$. nigrescens (green line) contains the bands of the stone background and additional ones that are due to the organic compounds of the biodeteriogen layers, by virtue of the features centred at 1340 and $1580 \mathrm{~cm}^{-1}$ of the D and $\mathrm{G}$ bands of carbon, respectively. The relative intensities of the latter bands, together with the broadening observed, suggest the presence of amorphous carbon in the biological crust. The intensity of the carbon bands decreases upon laser irradiation of the colonized sample at $1064 \mathrm{~nm}$ (red line), while the bands of the lithic substrate weakly emerge in the spectrum, indicating the partial removal of the biodeteriogen layer. Differently, the intensity of the Raman signal after irradiation at $355 \mathrm{~nm}$ (blue line) is extremely low, although a hint of weak carbon bands is detected in the corresponding zone of the spectrum. At this wavelength the biological layer is superficially damaged, as shown in Fig. $4 \mathrm{~b}$, and the sample surface does not display appreciable Raman response. These results provide evidence of the more efficient removal of the biological crust when operating the laser at $1064 \mathrm{~nm}$ in agreement with SEM-BSE observations, that signpost the mostly superficial effect of UV laser irradiation. It is also important to notice that the 
absence of new bands in the spectra of the irradiated zones provides evidence of chemical stability of the lithic substrate. Figure 8 also displays the spectrum of a colonized sample zone exposed to sequential IR-UV laser irradiation (magenta line). In this case the bands of bare dolomite feature a good signal to noise ratio, while amorphous carbon bands at 1340 and $1580 \mathrm{~cm}^{-1}$ fully disappear. Again, this is in correspondence with the SEM-BSE results and confirms that this mode of sequential irradiation entirely removes the layer of organic material while the substrate is preserved, as indicated by the presence of intense bands at $175,299,727$ and $1097 \mathrm{~cm}^{-1}$ attributed to the dolostone minerals.

\section{Discussion}

In this work we treated dolomite stone samples colonized by crustose lichens with sequences of IR (1064 nm) and UV (355 nm) nanosecond laser pulses and by the combined application of IR and UV pulses in sequential mode. At each wavelength the applied laser fluences were below the corresponding ablation thresholds of dolostone to ensure the preservation of the lithic substrate. The different laser irradiation schemes applied lead to various degrees of elimination and damage of epilithic and endolithic colonizers.

We have shown that irradiation with the IR laser wavelength on crust dominated by unhealthy Caloplaca sp. induces the extensive elimination of these epilithic thalli and considerable damage of endolithic microbial cells. This is in good agreement with previous findings [22] of removal and inactivation of the lichen $V$. nigrescens from the same lithotype and of damage of the internal microbiota community under similar IR laser conditions (fluence and number of pulses). However, the effects observed therein are more intense than in the previous study, probably due to the unhealthy state of the Caloplaca sp. 
thalli, and suggest that laser cleaning of epilithic colonization on monumental stone from polluted areas could be facilitated by the previous weakening of the lichen thalli. These observations open new strategies of treatment based in the combination of biocide and laser treatment [26]. Irradiation with $1064 \mathrm{~nm}$ Q-switched Nd:YAG laser has been investigated for the treatment of dermatopathogenical fungal colonies [27] and for inactivation of bacteria and yeasts [28]. The resulting inhibitory effects are discussed in relation with various mechanisms, including thermal damage, photochemical effects triggered by chromophore-induced absorption at the specific laser wavelength and photo mechanical effects associated with microcavitation phenomena and propagation of acoustic shock waves. In contrast, and according to our results, UV laser irradiation has proven to induce a rather superficial damaging effect in the lichen colonization crust. These differences are related with the higher light absorbance of $V$. nigrescens at $355 \mathrm{~nm}$ in comparison with that at $1064 \mathrm{~nm}$ [29]. Thus, light at $355 \mathrm{~nm}$ induces a superficial effect on the lichen crust, in contrast with the in-depth effect observed for IR laser treatment, by efficiently coupling to the upper lichen cortex and by leaving unaffected the endolithic colonizers. FT-Raman results support the stereomicroscopy and SEM-BSE observations showing a more efficient removal of microorganisms upon IR irradiation. Finally, the results herein show that the combination of the two wavelengths in the tested sequential laser irradiation protocol brings a clear advantage with respect to the single IR or UV wavelength irradiation modes. In this case the lichen upper cortex is totally or partially removed by the sequence of IR pulses and the exposed unprotected photobiont is eventually damaged by the following train of UV pulses. Again FT-Raman spectra of the doubly irradiated areas clearly display the unperturbed features of the lithic substrate, thus confirming removal of the biodeterioration layer under stone safeguard conditions. 
While these findings provide the framework for the efficient removal of crustose lichen on heritage dolomite stones, further works are in course devoted to test different laser irradiation protocols, using other wavelengths, pulse durations and pulse delivery modes, to optimize the removal of biodeteriogen layers on heritage stone substrates.

\section{Conclusions}

In this work we have investigated the use of Q-switched Nd:YAG laser delivering nanosecond pulses for the elimination of epilithic crustose lichen layers from heritage dolomite stone. Specifically, we compared different irradiation modes based on singlewavelength irradiation, at $1064 \mathrm{~nm}$ (IR) and at $355 \mathrm{~nm}$ (UV), and on IR-UV laser irradiation consisting in sequences of IR followed by UV pulses. We evaluated the structural and physiological damage inflicted on the microorganisms by laser irradiation, and assessed possible morphological and chemical changes on the treated substrate under the different laser irradiation conditions using stereomicroscopy, scanning electron microscopy with backscattered electron imaging and Fourier transform Raman spectroscopy. While single wavelength irradiation using IR or UV wavelengths led to the partial removal of the lichen crust and inflicted some degree of damage on endolithic microorganisms, it is demonstrated that the sequential IR-UV laser treatment ensures effective removal of lichen thalli and biological injuries of the endolithic microorganisms, like disruption of fungal lipid bodies and loss of content of fungal hyphae, thus providing a high degree of control of the biodeterioration processes of the lithic substrate and reducing the chances of lichen recolonization. The sequential dual irradiation procedure emerges as an advantageous approach for the laser removal of colonization crust on heritage stone substrates. 


\section{Acknowledgments}

Work funded by Program Geomateriales 2 (S2013/MIT-2914) financed by Comunidad de Madrid and Structural Funds (FSE and FEDER). M.O acknowledges contract by CSIC.

We are grateful to M. Furio, A. Jorge and L. Tormo from Servicio de Microscopia, MNCN-CSIC and to support by Red de Ciencia y Tecnología para la Conservación del Patrimonio Cultural (Ministerio de Ciencia e Innovación, Spain, Acción Complementaria HAR2010-11432-E). 


\section{References}

[1] Lasers in the preservation of cultural heritage. Principles and applications, C. Fotakis, D. Anglos, V. Zafiropulos, S. Georgiou and V. Tornari (Eds.), Taylor \& Francis, 2007.

[2] Lasers in the Conservation of Artworks, M. Castillejo, P. Moreno, M. Oujja, R. Radvan, J. Ruiz (Eds.), CRC Press, 2008.

[3] M. Oujja, E. Rebollar, M. Castillejo, C. Domingo, C. Cirujano, F. Guerra-Librero, Laser cleaning of terracotta decorations of the portal of Palos of the Cathedral of Seville, J. Cult. Herit., 6 (2005) 321-327.

[4] P. Pouli, M. Oujja, M. Castillejo, Practical issues in laser cleaning of stone and painted artefacts: optimisation procedures and side effects, Appl. Phys. A, 106 (2012) 447-464.

[5] S. Siano, J. Agresti, I. Cacciari, D. CIofini, M. Mascalchi, I. Osticioli, A.A. Mencaglia, Laser cleaning in conservation of stone, metal and painted artifacts: state of the art and new insights on the use of Nd:YAG lasers, Appl. Phys. A, 106 (2012) 419-446.

[6] P. Maravelaki-Kalaitzaki, V. Zafiropulos, C. Fotakis, Excimer laser cleaning of encrustation on Pentelic marble: procedure and evaluation of the effects, Appl. Surf. Sci., 148 (1999) 92-104.

[7] A. de Cruz, M.L. Wolbarsht, R.A. Palmer, S.E. Pierce, E. Adamkiewicz, Er:YAG laser applications on marble and limestone sculptures with polychrome and patina surfaces, $\mathrm{J}$. Cult. Herit., 5 (2005) 113-124.

[8] G. Marakis, P. Pouli, V. Zafiropulos, P. Maravelaki-Kalaitzaki, Comparative study on the application of the 1st and the 3rd harmonic of a Q-switched Nd:YAG laser system to clean black encrustation on marble, J. Cult. Herit., 4 (2003) 83s-91s. 
[9] P. Pouli, C. Fotakis, B. Hermosin, C. Saiz-Jimenez, C. Domingo, M. Oujja, M. Castillejo, The laser-induced discoloration of stonework; a comparative study on its origins and remedies, Spectrochim. Acta A, 71 (2008) 932-945.

[10] S.S. Potgieter-Vermaak, R.H.M. Godoi, R.V. Grieken, J.H. Potgieter, M. Oujja, M. Castillejo, Micro-structural characterization of black crust and laser cleaning of building stones by micro-Raman and SEM techniques, Spectrochim. Acta A, 61 (2005) 2460-2467.

[11] G. Caneva, M.P. Nugari, O. Salvadori, Control of biodeterioration and bioremediation techiques in: G. Caneva, M.P. Nugari, O. Salvadori (Eds.) Plant biology for cultural heritage: biodeterioration and conservation Getty Conservation Institute, Los Angeles 2008, pp. 309-346.

[12] E. Doehne, C. Price, Stone Conservation: An Overview of Current Research, in: T.G.C. Institute (Ed.), Los Angeles, CA, 2010, pp. 164.

[13] K. Sterflinger, G. Piñar, Microbial deterioration of cultural heritage and works of arttilting at windmills, Appl. Microbiol. Biotechnol., 97 (2013) 9637-9646.

[14] C. Ascaso, J. Wierzchos, R. Castelló, Study of the biogenic weathering of calcareous litharenite stones caused by lichen and endolithic microorganisms, Int. Biodeter. Biodegrad., 42 (1998) 29-38.

[15] C. Ascaso, J. Wierzchos, V. Souza-Egipsy, A. de los Rios, J.D. Rodrigues, In situ evaluation of the biodeteriorating action of microorganisms and the effects of biocides on carbonate rock of the Jeronimos Monastery (Lisbon), Int. Biodeter. Biodegrad., 49 (2002) $1-12$.

[16] M. Bartolini, A.M. Pietrini, S. Ricci, Use of UV-C irradiation on artistic stone works for control of algae and cyanobacteria, in P.M. Tiano, G. (Ed.) Microbes and Art: The role 
of microbial communities in the degradation and protection of cultural heritage, CNR, Florence, 1999, pp. 221-227.

[17] B. Cámara, A. de los Ríos, M. Urizal, M. Álvarez de Buergo, M.J. Varas, R. Fort, C. Ascaso, Characterizing the Microbial Colonization of a Dolostone Quarry: Implications for Stone Biodeterioration and Response to Biocide Treatments, Microbial Ecology, 62 (2011) 299-313.

[18] A. de los Ríos, S. Pérez-Ortega, J. Wierzchos, C. Ascaso, Differential effects of biocide treatments on saxicolous communities: case study of the Segovia cathedral cloister (Spain), Int. Biodeter. Biodegrad., 67 (2012) 64-72.

[19] E. Sarantopoulou, Z. Kollia, I. Gomoiu, Preventing biological activity of Ulocladium sp spores in artifacts using 157 nm laser, Appl. Phys. A, 83 (2006) 663-668.

[20] A. de Cruz, M.L. Wolbarsht, A. Andreotti, M.P. Colombini, D. Pinna, C.F. Culberson, Investigation of the Er:YAG laser at $2.94 \mu \mathrm{m}$ to remove lichens growing on stone, Stud. Conserv., 54 (2009) 268-277.

[21] A.J. López, T. Rivas, J. Lamas, A. Ramil, A. Yáñez, Optimisation of laser removal of biological crusts in granites, Appl. Phys. A, 100 (2010) 733-739.

[22] M. Speranza, M. Sanz, M. Oujja, A. de los Rios, J. Wierzchos, S. Pérez-Ortega, M. Castillejo, C. Ascaso, Nd-YAG laser irradiation damages to Verrucaria nigrescens, Int. Biodeter. Biodegrad., 84 (2013) 281-290.

[23] I. Osticioli, M. Mascalchi, D. Pinna, S. Siano, Removal of Verrucaria nigrescens from Carrara marble artefacts using Nd:YAG lasers: comparison among different pulse durations and wavelengths, Appl. Phys. A, 118 (2015) 1517-1526. 
[24] J. Wierzchos, C. Ascaso, Application of back-scattered electron imaging to the study of the lichen-rock interface, J. Microsc., 175 (1994) 54-59.

[25] S. Gurasekaram, G. Anbalagan, S. Pandi, Raman and IR spectra of carbonate of calcite structure, J. Raman Spectrosc., 37 (2006) 892-899.

[26] A. de los Ríos, S. Pérez-Ortega, M.A. Martínez-Fernández, V. Galván, M. Speranza, M. Oujja, M. Sanz, M. Castillejo, J. Wierzchos, E, Pérez-Montserrate, M. Alvarez de Buergo, R. Fort, G. Hualparimachi , F. Astete, C. Ascaso, Biodeterioration and conservartion of Historic Sanctuary of Machu Picchu, Book of Abstracts, p. 58, XVI International Biodeterioration and Biodegradation Symposium (IBBS 16), Lodz, Poland, 35 September 2014.

[27] E. Vural, H.L. Winfield, A.W. Shingleton, T.D. Horn, G. Shafirstein, The effects of laser irradiation on Trichophyton rubrum growth, Lasers Med. Sci., 23 (2008) 349-353.

[28] G.D. Ward, I.A. Watson, D.E. Stewart-Tull, A.C. Wardlaw, C.R. Chatwin, Inactivation of bacteria and yeasts on agar surfaces with high power Nd:YAG laser light, Lett. Appl. Microbiol., 23 (1996) 136-40.

[29] J.A. Rojas-Fernández, A. Balza-Quintero, V. Marcano, P. Andrea Rojas, D. DávilaVera, Z. Peña-Contreras, R.V. Mendoza-Briceño, E. Palacios-Prü, Metabolitos secundarios de líquenes de la zona nival de la Sierra Nevada de Mérida-Venezuela y su papel en la absorción de la radiación ultravioleta, Anales del Jardín Botánico de Madrid Vol. 65 (2008) 59-72. 


\section{Figure captions}

Figure 1. a) Steromicroscopy image of an area of the dolostone sample from Segovia irradiated with 100 pulses of $1064 \mathrm{~nm}$ at $2.5 \mathrm{Jcm}^{-2}$. b) SEM-BSE image of unhealthy Caloplaca sp. epilithic thalli and endolithic colonization showing a general view at the boundary between the non-irradiated (right of the arrow) and the irradiated area (left of the arrow).

Figure 2. SEM-BSE images of Caloplaca sp. epilithic thalli and endolithic colonization of the dolostone sample from Segovia. a) Detail of the non-irradiated unhealthy lichen thallus, showing the upper cortex (asterisk) and the photobiont layer (between the two dotted lines). b) View of a laser-irradiated area (100 pulses of $1064 \mathrm{~nm}$ at $2.5 \mathrm{Jcm}^{-2}$ ) showing partially removed epilithic thalli (arrow) and damaged microbial cells localized near the surface (black empty spaces revealing to the absence of cellular protoplasts).

Figure 3. a) Stereomicroscopy image of a fragment of the dolomite sample from Redueña quarry covered by the dark-brown areolate thalli of $V$. nigrescens before laser irradiation. b) SEM-BSE image of the $V$. nigrescens thalli and endolithic colonization of a nonirradiated area of the sample. The upper cortex is marked by asterisks, the photobiont layer by a thin long arrow and the medulla by a wide short arrow. The square frames a zone shown in Fig. 4a with higher magnification.

Figure 4. a) SEM-BSE image of the dolomite sample from Redueña quarry showing detail of an areole of $V$. nigrescens (in the area within the square of Fig. 3b). The upper cortex is indicated by an asterisk and the photobiont layer appears between thin white arrows. b) Stereomicroscopy image of the thalli of $V$. nigrescens after irradiation with 100 pulses of $0.5 \mathrm{Jcm}^{-2}$ at $355 \mathrm{~nm}$. The rectangle marks an area from where SEM-BSE image of Fig. 5a was taken. 
Figure 5. SEM-BSE images of the $V$. nigrescens thalli and endolithic colonization after irradiation with 100 pulses of $0.5 \mathrm{Jcm}^{-2}$ at $355 \mathrm{~nm}$. a) Image acquired in the zone marked by a rectangle in Fig. 4b, displaying areoles damaged by laser irradiation (arrows). b) Irradiated areole at higher magnification showing the partial removal of the upper cortex (arrow) and plasmolized fungal cortex cells (asterisks).

Figure 6. a) Stereomicroscopic image of thalli of $V$. nigrescens colonizing Redueña quarry dolostone sample after being subjected to sequential IR-UV laser treatment with 100 pulses of $1064 \mathrm{~nm}$ at $2.5 \mathrm{~J} \mathrm{~cm}^{-2}$ followed by 100 pulses of $355 \mathrm{~nm}$ at $0.5 \mathrm{Jcm}^{-2}$. b) SEM-BSE image of an area of the sample, within the dotted line of image a), showing how lichen thalli that have been removed by laser treatment (asterisks) and how endolithic fungal hyphae are almost completely destroyed (arrows).

Figure 7. SEM-BSE images of $V$. nigrescens colonizing Redueña quarry dolostone sample after sequential IR-UV laser treatment with 100 pulses of $1064 \mathrm{~nm}$ at $2.5 \mathrm{~J} \mathrm{~cm}^{-2}$ followed by 100 pulses of $355 \mathrm{~nm}$ at $0.5 \mathrm{Jcm}^{-2}$. a) Partially ablated lichen areoles within the irradiated area. b) Higher magnification image of the areoles of image a). The thin arrow points to detachments of the upper cortex and the wide arrow marks the position of exposed unprotected algae by loss of the upper cortex. The asterisks above black spaces mark exposed algal cells that have experienced loss of some of their content.

Figure 8. FT-Raman spectra of the dolomite stone sample from Redueña quarry. The black line corresponds to the spectrum of the bare stone, and the green one to that taken on a sample surface colonized by epilithic crustose lichen $V$. nigrescens before laser irradiation. The red line is the spectrum of the colonized sample after laser irradiation with 100 pulses of $2.5 \mathrm{~J} / \mathrm{cm}^{2}$ at $1064 \mathrm{~nm}$ and the blue line corresponds to the sample irradiated with 100 pulses of $0.5 \mathrm{~J} / \mathrm{cm}^{2}$ at $355 \mathrm{~nm}$. Finally, the magenta line is the spectrum of an area treated 
with a sequence of 100 IR pulses followed by $100 \mathrm{UV}$ pulses at the given fluences. The wavenumbers of the assigned bands are indicated. 

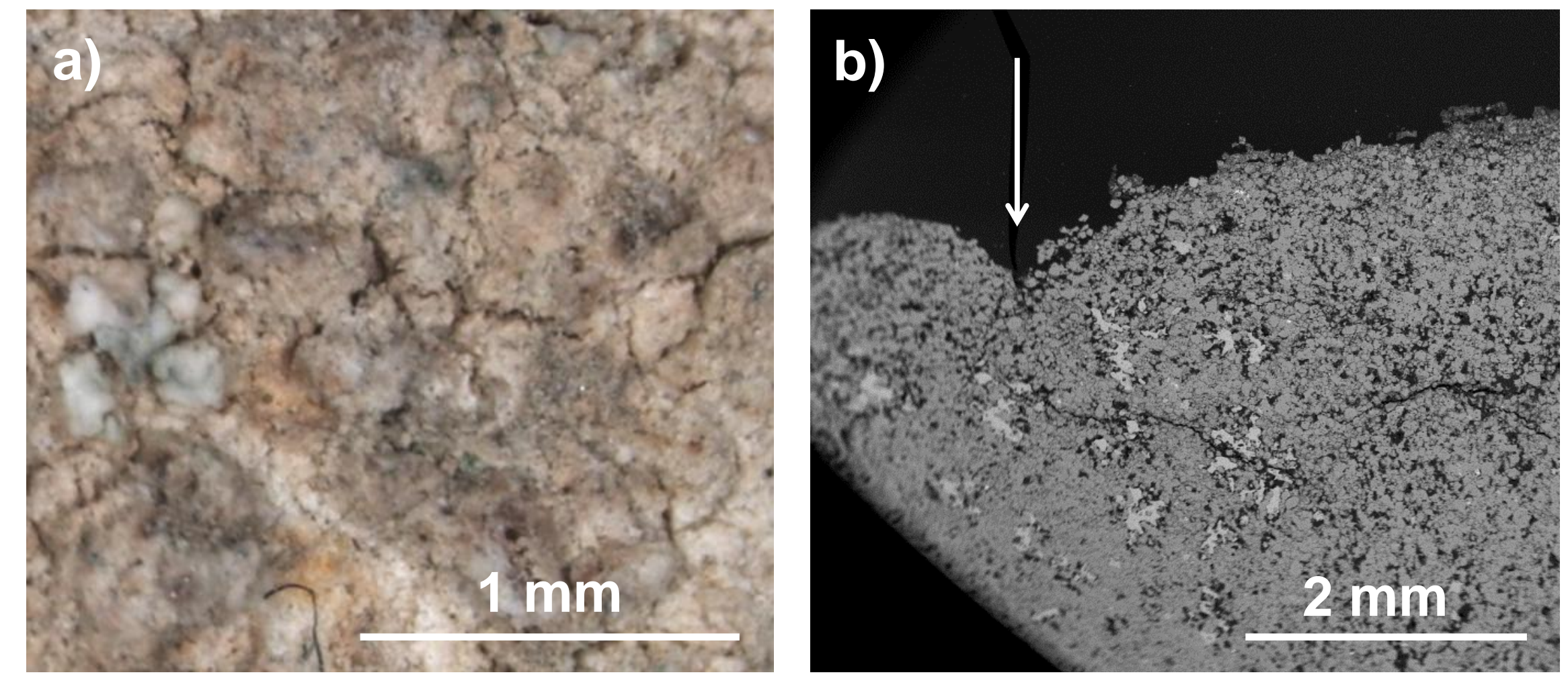

\section{Figure 1}



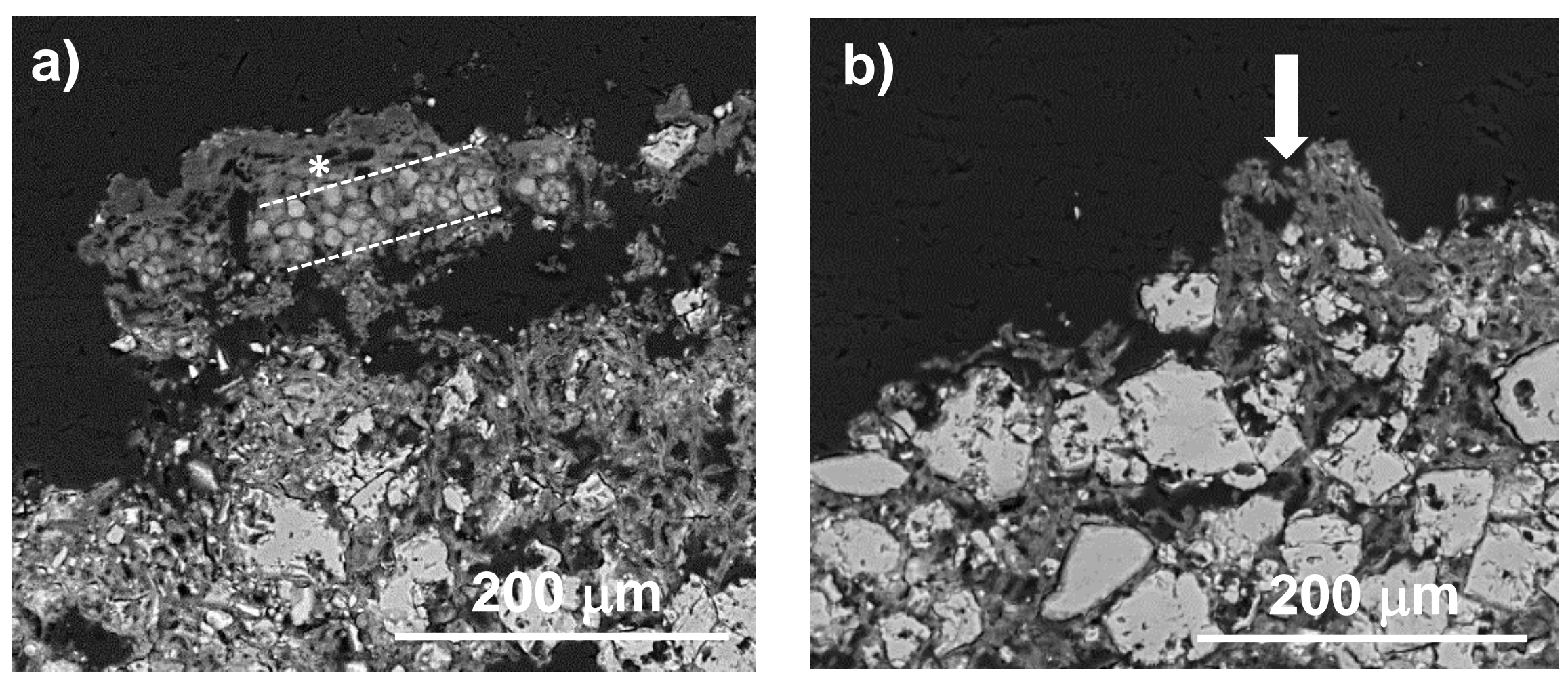

Figure 2 

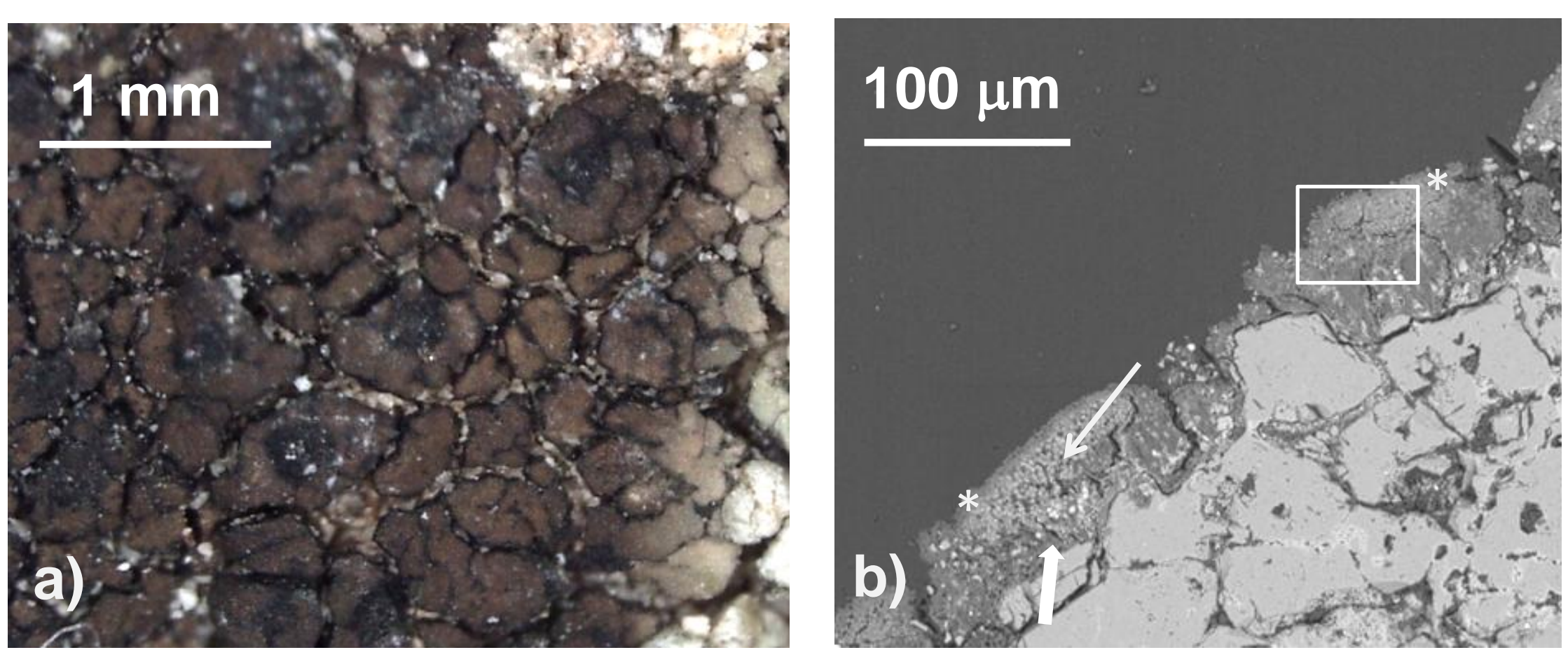

\section{Figure 3}



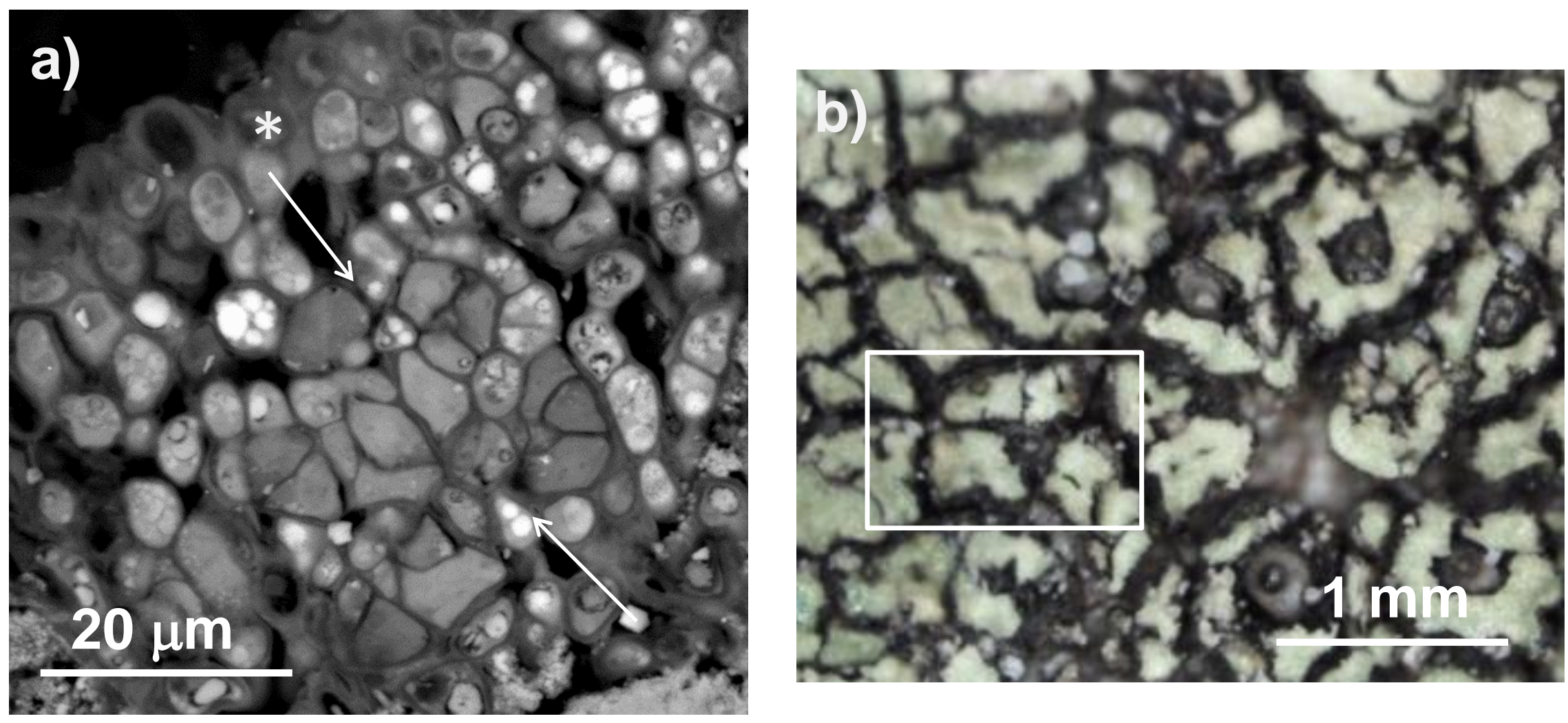

Figure 4 
Figure

Click here to download Figure: Figure5R.pptx
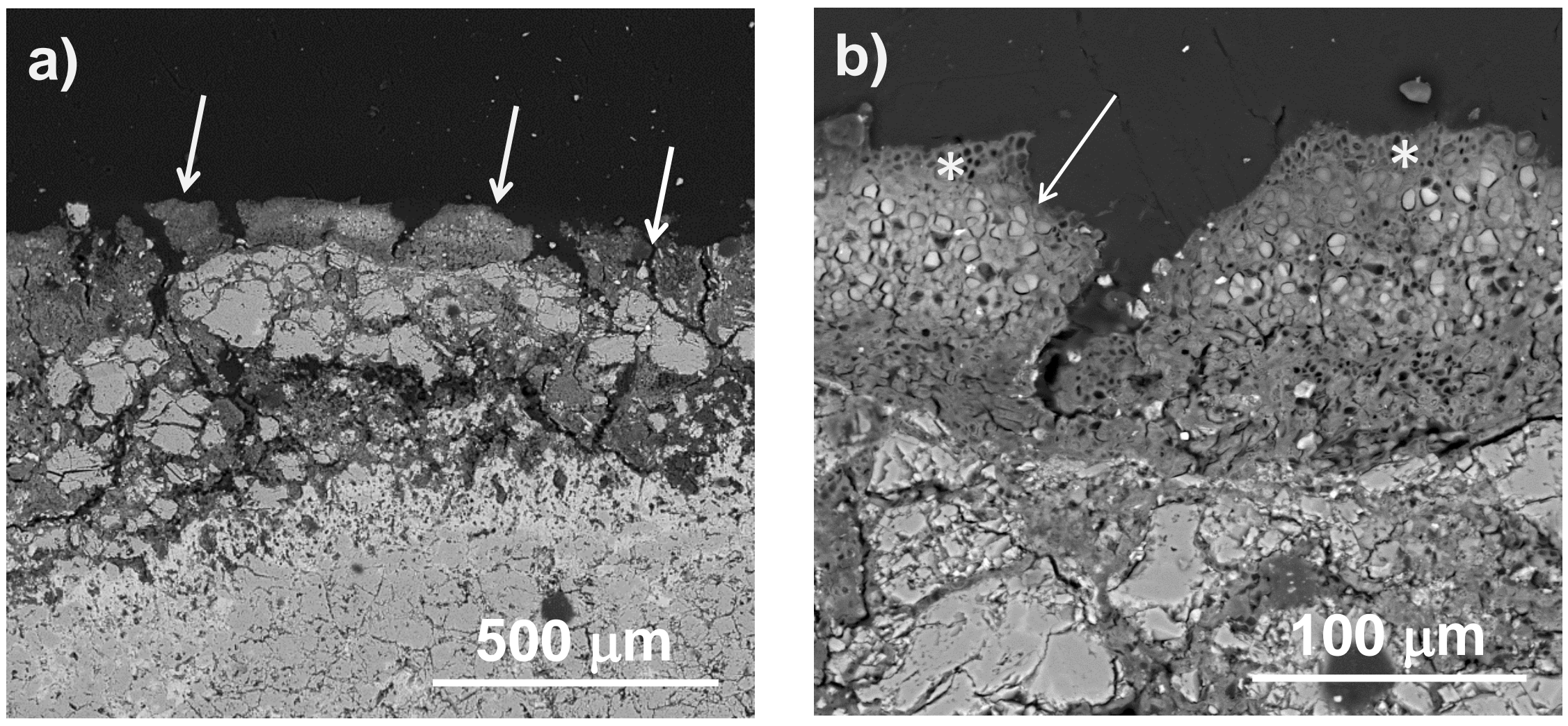

Figure 5 

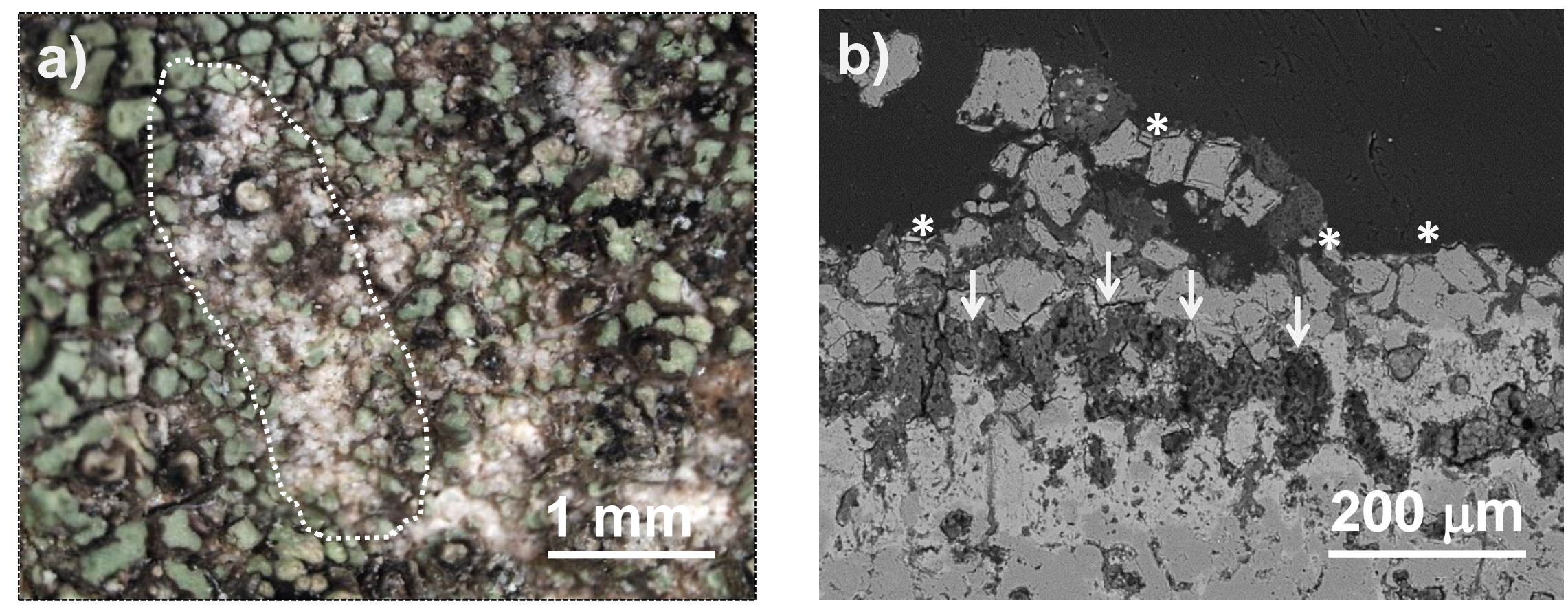

Figure 6 

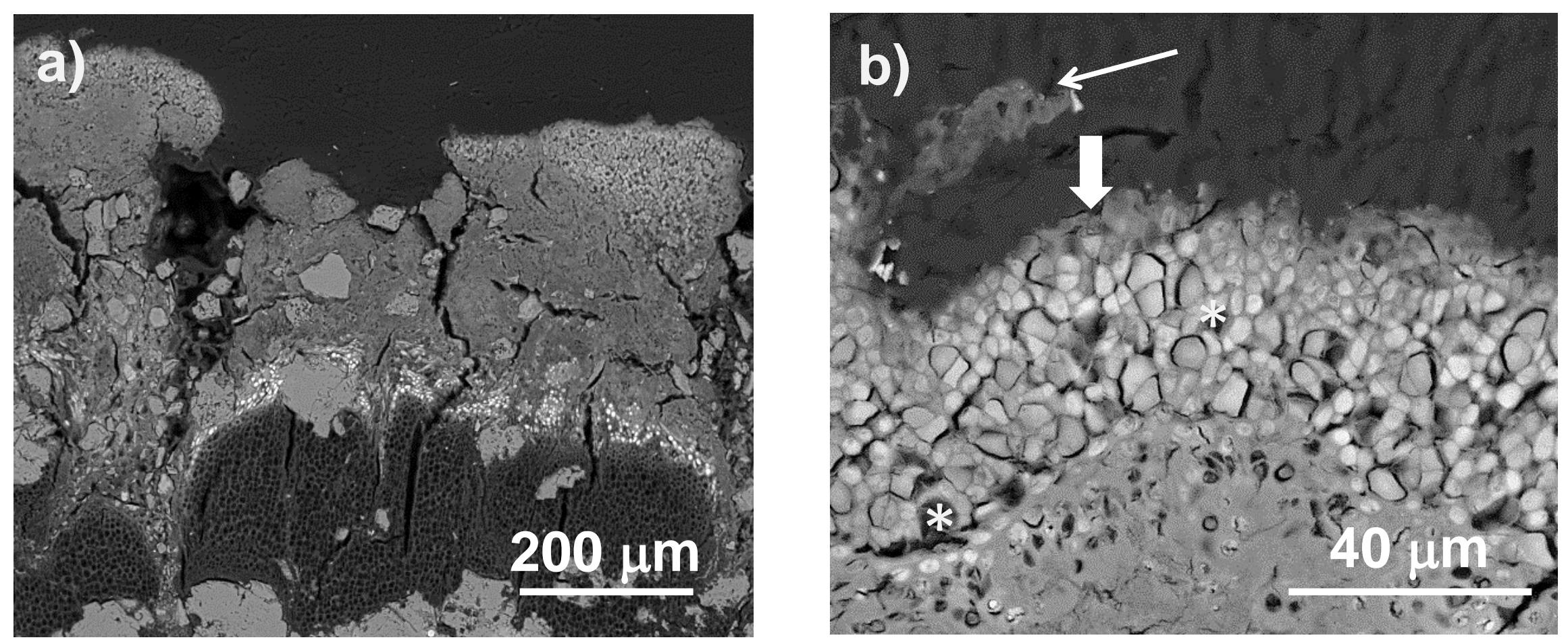

Figure 7 


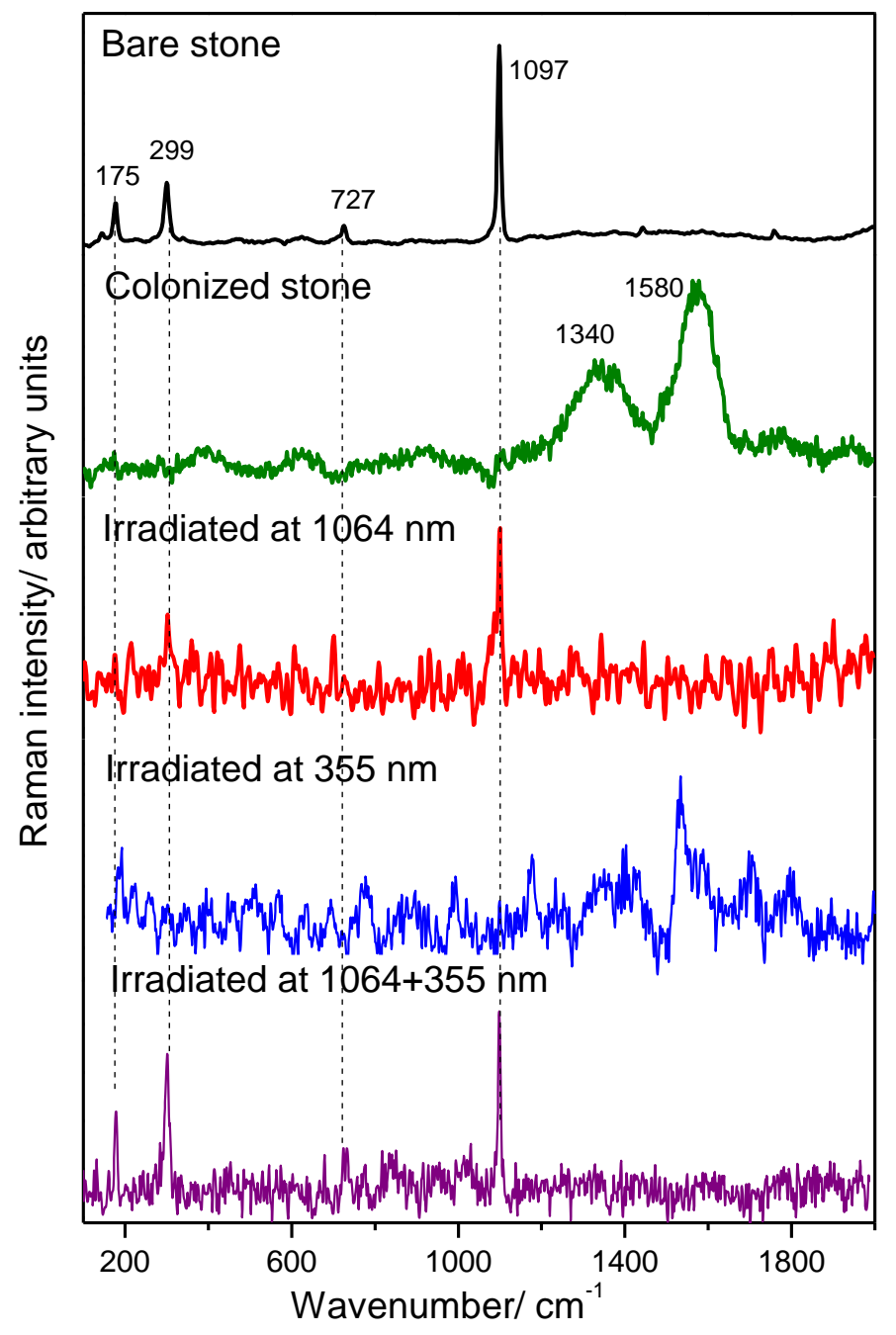

Figure 8 